\title{
Cardiac Arrest Secondary to Short-Coupled TdP: A Case Report
}

\author{
Kamal Preet Kaur ${ }^{1 *}$ and Niels Eske Bruun ${ }^{1,2,3}$ \\ ${ }^{1}$ Department of Cardiology, Zealand University Hospital, Roskilde, Denmark \\ ${ }^{2}$ Department of Clinical Medicine, Faculty of Health and Medical Sciences, University of Copenhagen, Denmark \\ ${ }^{3}$ Clinical Institute, Aalborg University, Aalborg, Denmark
}

*Corresponding author: Kamal Preet Kaur, Department of Cardiology, Zealand University Hospital, Roskilde, Denmark.
Received Date: October 22, 2019

Published Date: October 29, 2019

\begin{abstract}
Background: The short-coupled variant of Torsade de Pointes (Sc-TdP) is rare and causes Torsade-like polymorphic ventricular tachycardia (VT) without the classical QT-prolongation. It is initiated by a remarkably short coupling interval $(<300 \mathrm{~ms})$. The arrhythmia is important to identify as the treatment differs from another traditional polymorphic VT.

Case summary: A 76 years old male was transferred to our coronary care unit after surviving an out of hospital cardiac arrest. Apart from frequent premature ventricular beats, the electrocardiogram was normal. In the acute phase, left ventricular function was decreased, but quickly normalized. The coronary angiography (CAG) revealed normal coronary arteries. The first night after the CAG was performed, the patient experienced a new episode of cardiac arrest with successful resuscitation. The telemetry revealed Sc-Tdp. He was initially treated with intravenous amiodarone, later changed to oral metoprolol. An implantable cardioverter defibrillator (ICD) was inserted, metoprolol was replaced by verapamil and the patient was discharged. A 3-month follow-up visit revealed two episodes of short-lasting VT but no therapy was delivered by the ICD.

Conclusion: Data on the clinical management of patients with Sc-TdP during the acute phase and long-term clinical outcome remain minimal. In our present case, verapamil seems to be an effective drug in suppressing the arrhythmias, but it does not eliminate the need of an ICD to reduce the risk of sudden cardiac death.
\end{abstract}

Keywords: Short-coupled tdp, Sudden cardiac death, Verapamil

\section{Introduction}

Torsade de Pointes (TdP) is the most common form of polymorphic ventricular tachycardia (VT) that occurs in the setting of an abnormally long QT interval. The short-coupled variant of TdP (Sc-TdP) is rare and causes Torsade-like polymorphic VT without the classical QT-prolongation. It is associated with a high incidence of sudden cardiac death. The electrocardiogram (ECG) displays typical TdP pattern but it is initiated by remarkably short coupling interval ( $<300 \mathrm{~ms}$ ) of the first beat or of the isolated premature beats [1$3]$. The premature beats have the same morphology suggesting one focal origin. The literature describes healthy young patients with a family history of sudden cardiac death but the responsible gene(s) have not yet been resolved though a few cases have revealed RyR2 mutation [5]. Data on the clinical management of patients with ScTdP during the acute phase and long-term clinical outcome remain minimal. Only isolated cases have been published since the initial description by Leenhardt et al. in 1994 [6]. We report a case of a 76 years old male with the absence of structural heart disease who survived an in hospital cardiac arrest secondary to this kind of malignant arrhythmia.

\section{Case Presentation}

In September 2018, a 67 years old male with a history of hypertension, hypercholesterolemia, Chronic Obstructive Pulmonary Disease (COPD), previous catheter ablation for atrioventricular nodal re-entrant tachycardia, paroxysmal atrial fibrillation (treated with Metoprolol and Digoxin) and no family history of sudden death was admitted to hospital after experiencing an out of hospital cardiac arrest, while being in a market. Bystander cardiopulmonary resuscitation (CPR) was initiated immediately. Emergency medical services arrived within 3 minutes after the initiation of CPR and found that the patient had ventricular 
fibrillation (VF). The patient was successfully converted to sinus rhythm after receiving 2 shocks, gained return of spontaneous circulation (ROSC) and was transported to the local emergency department (ED). Upon arrival, the patient was found conscious and stable. He denied having any symptoms prior to his collapse. A bedside transthoracic echocardiography (TTE) revealed a left ventricle ejection fraction (LVEF) of $35-40 \%$ and anterior hypokinesia. The electrocardiogram (ECG) showed a sinus rhythm without any significant changes (Figure 1) and no QT-prolongation. Extensive laboratory studies revealed Troponin I up to $641 \mathrm{ng} / \mathrm{L}$. The treatment for Non-ST-segment elevation myocardial infarction was initiated following the national guidelines. The patient was transferred to our coronary care unit the following day, where a coronary arteriography (CAG) was performed. The CAG revealed normal coronary arteries with non-significant atheromatosis in the right coronary artery. A CT-thorax was ordered to exclude a pulmonary embolism. The first night after the CAG was performed, the patient experienced a new episode of cardiac arrest. An external defibrillator was immediately attached to the patient and one shock was delivered due to shockable rhythm, re-establishing circulation. Upon arrival of the resuscitation team, the patient had ROSC. Since the patient's previous ECGs had revealed a normal QT-interval, the patient was initially treated with intravenous Magnesium and Amiodarone bolus followed by continuous infusion as per the guidelines. New bedside TTE was performed showing a LVEF of 40 \%. The ECG (Figure 3) showed sinus tachycardia with normal QT-interval and no signs of ischemia. The telemetry (Figure 2) was reviewed demonstrating a polymorphic VT, which was similar to TdP in morphology. The coupling interval of the initiating premature beat to the first beat of the polymorphic VT was $<240 \mathrm{~ms}$. Before the initiation of the Sc-TdP the patient ran premature ventricular contractions (PVC) as in bigeminal rhythm, 100 beats per minute (bpm), excluding that the TdP was triggered by bradycardia.

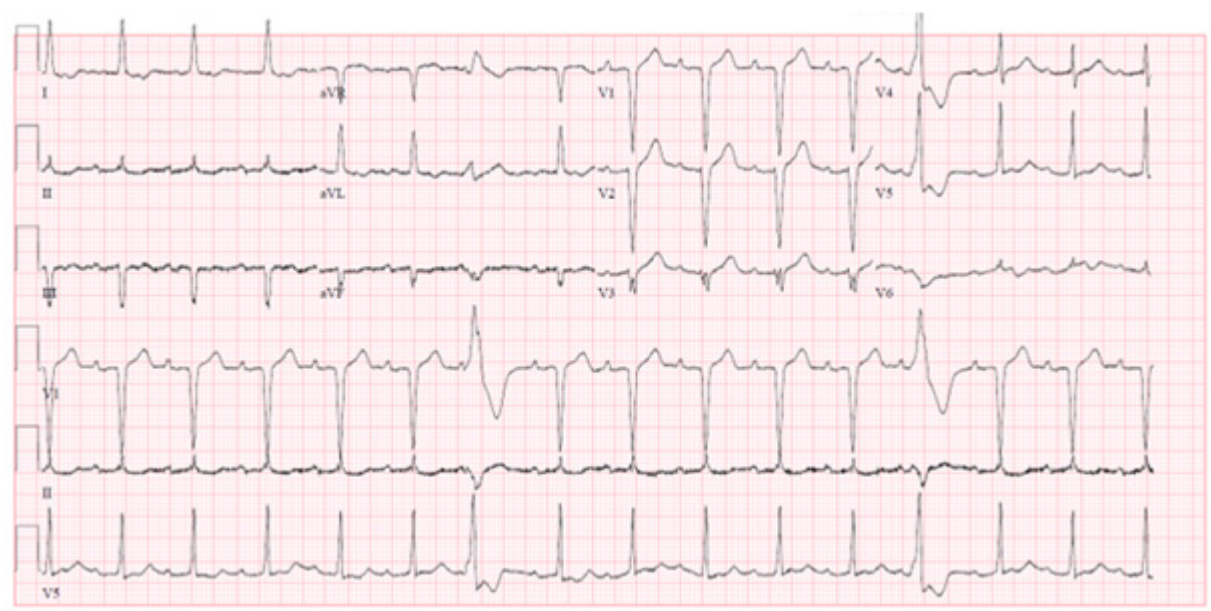

Figure 1: Twelve-lead ECG during sinus rhythm. Note the normal durations of QT interval (386 ms) and QTc interval (474 ms) interval at a mean heart rate of 91 beats/min. The premature ventricular contractions look similar in morphology suggesting the same origin. Paper speed $25 \mathrm{~mm} / \mathrm{s}$.

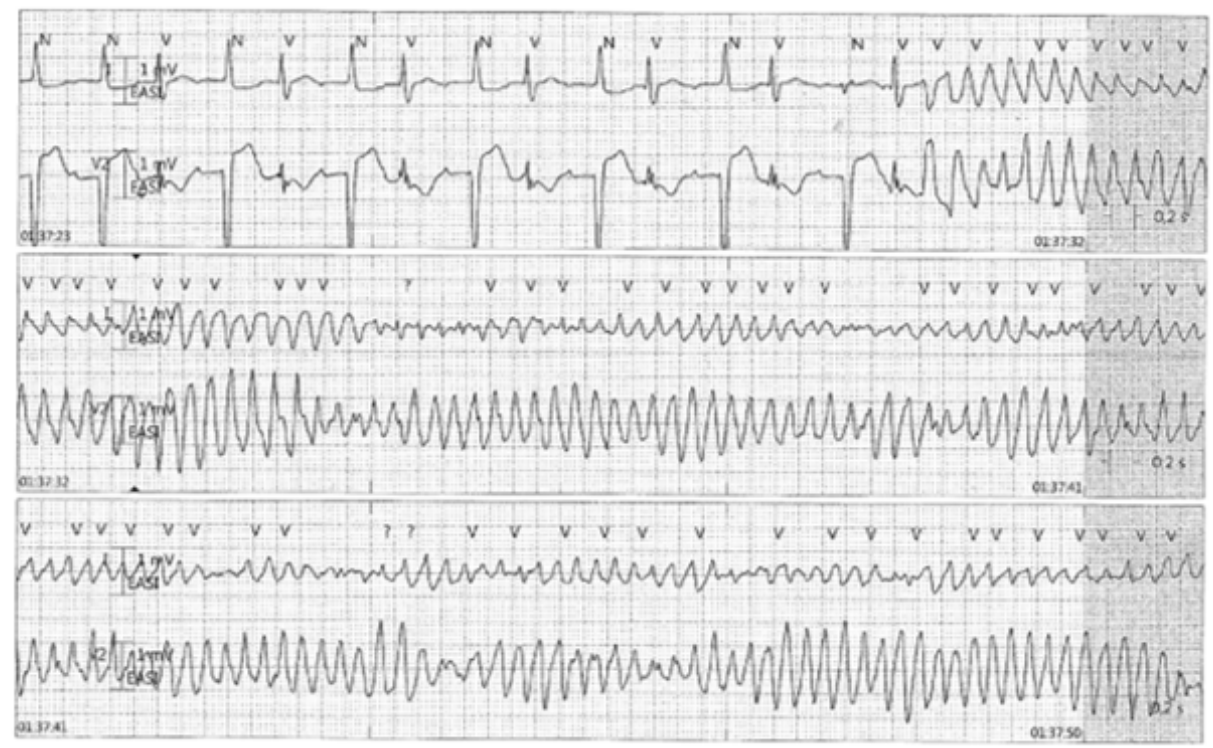

Figure 2: Short-coupled torsade de pointes (TdP) degenerating into ventricular fibrillation with a coupling interval of <240 ms of the first ventricular beat initiating TdP and leading to cardiac arrest. Prior to the polymorphic ventriucular tachycardia, there is a bigeminal rhythm. 


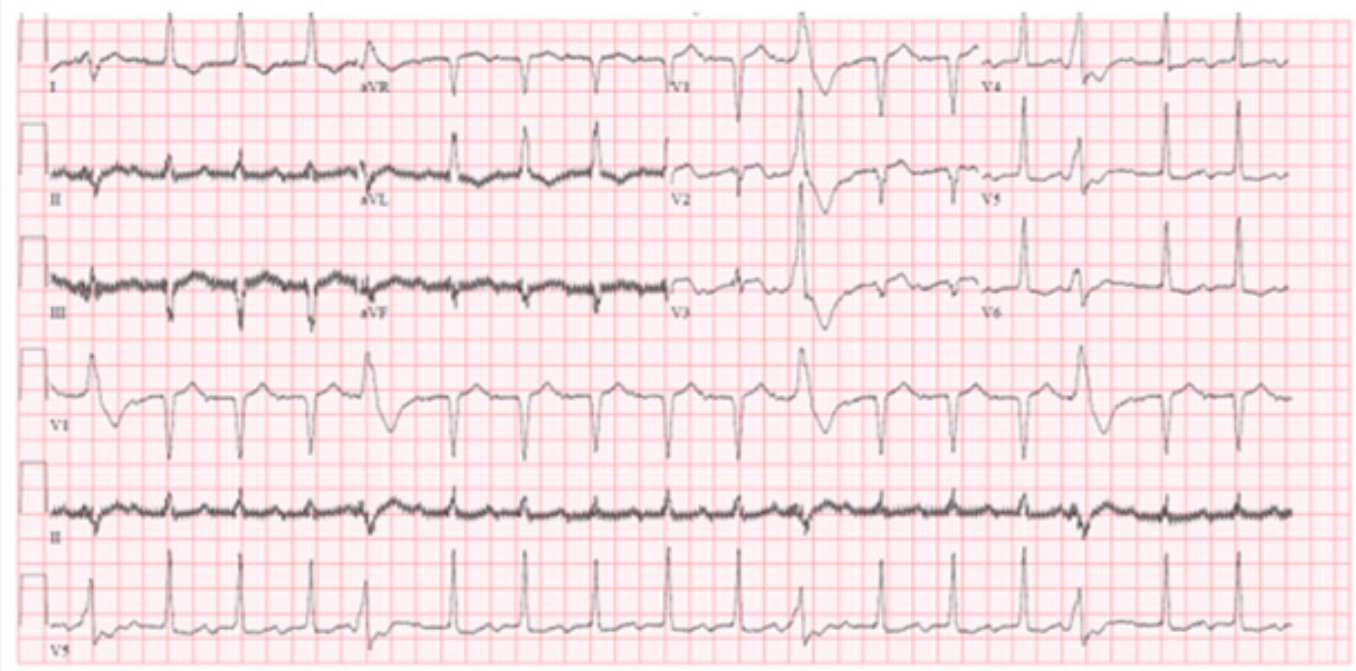

Figure 3: Twelve-lead ECG during sinus rhythm recorded some hours after the cardiac arrest. Note the normal durations of QT interval (332 $\mathrm{ms}$ ) and QTc interval (436 ms) interval at a mean heart rate of 104 beats/min. Premature ventricular contractions with same morphology. Paper speed $25 \mathrm{~mm} / \mathrm{s}$.

An implantable cardioverter defibrillator (ICD) was inserted and Metoprolol was replaced by Verapamil $120 \mathrm{mg}$ twice a day as suggested by the ESC guidelines, and the patient was discharged. During the first month, the remote monitoring system revealed a few episodes of 8-10 beats with non-sustained VT (NSVT), where no therapy was delivered. LVEF was normalized. The dose of Verapamil was increased to a dose of $240 \mathrm{mg}$ in the morning and $120 \mathrm{mg}$ in the evening with no further events the next month. Three month later, the remote monitoring system notified two episodes of short-lasting VT (polymorphic VT and NSVT), no therapy delivered by the ICD. Extensive laboratory tests were normal. The patient is currently receiving Verapamil $360 \mathrm{mg}+0 \mathrm{mg}+120 \mathrm{mg}$. Since then, no new incident of arrhythmia has been reported.

\section{Discussion}

In 1994 Leenhardt et al. described a new variant of polymorphic VT triggered by premature ventricular contractions (PVC) characterized by an extremely short-coupling interval (<300 ms), named the short-coupled variant of Torsade de Pointes [1]. Not long ago, a systematic review of case reports with Sc-TdP by Almehairi et al. suggested that a coupling interval $<400 \mathrm{~ms}$ was the most reliable measurement (sensitivity 100\%, specificity 97\%) [6]. In recent years, individual case reports have indicated the importance of identifying the Sc-TdP accurately and early as the initial clinical treatment and the long-term management differs from those of another traditional VT [2-6]. Verapamil, a calcium channel blocker, has been reported to be an effective drug in suppressing the arrhythmias in some patients with Sc-TdP, while drugs such as $\beta$-blockers and Amiodarone have not proven to be effective as a long-term treatment [1-6]. Leenhardt et al. reported a series of 14 patients with Sc-TdP [1]. Among the 14 patients, 2 were treated with $\beta$-blockers and died suddenly. Among the rest, 3 patients got an ICD ( 2 of them received Verapamil at the same time), whereas the others were treated with Verapamil solely. There were no deaths reported in the group with ICD. Post tracing studies revealed that all 3 patients with ICD experienced defibrillation shocks, which were delivered appropriately, during a follow-up of 4-7 years. In the group treated with Verapamil alone, a 7-year follow up revealed 2 sudden deaths, while the rest were alive but had persistent short-coupled ventricular premature beats. Yanfei et al. reported three cases with Sc-TdP [2]. All 3 patients were discharged with Verapamil. Patient 1 stopped taking Verapamil and died suddenly a year after being discharged, whereas the remaining two patients continued to take Verapamil and stayed alive. These cases indicate that Verapamil is not effective enough to eliminate the need of an ICD to reduce the risk of sudden cardiac death $[1,4,6]$.

A Sc-TdP with PVCs having the same morphology suggest one focal origin; therefore, a catheter ablation is considered as an additional option in patients with a high number of PVC or repeated episodes of VT/VF $[3,6]$. There are few studies reporting ablation of PVC to reduce the incidence of Sc-TdP. Haïssaguerre et al [3]. showed that PVCs originating from the distal Purkinje system triggered polymorphic VT in patients without structural heart disease. The study revealed successful suppression of PVCs with radiofrequency ablation, indicating a clinical benefit [3]. The literature does not provide convincing evidence of an association between specific genes and the occurrence of Sc-TdP. In one study Cheung et al. found the RyR2-H29D mutation to be associated with a case of Sc-TdP at rest [5]. A functional analysis demonstrated that RyR2-H29D causes a leaky channel at diastolic levels for Ca2+ under non-stress conditions. However, no further genes have been identified and this is an area, which requires further research. The genetic testing was not performed in our patient, since he did not present with a family history of sudden cardiac death. The present case is an example of how life-threatening an arrhythmia Sc-TdP is, if it is not identified and treated immediately. However, the area needs further investigation to provide the patients a better prevention, acute treatment and long-term management. 


\section{Acknowledgement}

The authors confirm that written consent for submission and publication of this case report including images and associated text has been obtained from the patient in line with COPE guidance.

\section{Conflict of Interest}

No conflict of interest.

\section{References}

1. Leenhardt A, Glaser E, Burguera M, Nürnberg M, Maison-Blanche P, et al. (1994) Short-coupled variant of torsade de pointes. A new electrocardiographic entity in the spectrum of idiopathic ventricular tachyarrhythmias. Circulation 89: 206-215.

2. Yanfei R, Lin W (2001) Short coupled variant of torsade de pointes. J Tongji Med Univ 21:30-31.
3. Haïssaguerre M, Shoda M, Jaïs P, Nogami A, Shah DC, et al. (2002) Mapping and ablation of idiopathic ventricular fibrillation. Circulation J 106: 962-967.

4. Chiladakis JA, Spiroulias G, Koutsogiannis N, Zagli F, Alexopoulos D, et al. (2008) Shortcoupled variant of Torsade de Pointes as a cause of electrical storm and aborted sudden cardiac death: insights into mechanism and treatment. Hellenic J Cardiol 49(5): 360-364.

5. Cheung JW, Meli AC, Xie W, Mittal S, Reiken S, et al. (2015) Short-coupled polymorphic ventricular tachycardia at rest linked to a novel ryanodine receptor (RyR2) mutation: leaky RyR2 channels under non-stress conditions. Int J Cardiol 180: 228-236.

6. Mohammed Almehairi, Alawi A Alshiekh-Ali, Ahmed Alfagih (2018) Idiopathic short-coupled ventricular tachyarrhythmias: Systematic review and validation of electrocardiographic indices. Egypt Heart J 70(4): 301-306. 